\title{
Perfil clínico e terapêutico de pacientes internados com úlceras de membros inferiores
}

\section{Clinical and therapeutic profile of patients insid with ulcers of inferior members}

\author{
Antônio Belmiro Peixôto Júnior' • Alana Tamar Oliveira de Sousa ${ }^{2} \bullet$ Lidiane Lima de Andrade ${ }^{3}$ \\ Matheus Figueiredo Nogueira ${ }^{4}$
}

\begin{abstract}
RESUMO
Objetivo: Descrever o perfil sociodemográfico e clínico de pacientes internados com úlceras de membros inferiores em um hospital escola; identificar os principais procedimentos clínicos e cirúrgicos realizados para o tratamento desses pacientes e relacionar os fatores de risco e as complicações encontradas nessas feridas. Método:Trata-se de uma pesquisa documentaldescritiva, com abordagem quantitativa, desenvolvida em um hospital escola, no município de Campina Grande-PB, por meio da análise de prontuários de pacientes que estiveram internados nas clínicas médicas, no período de junho de 2012 a março de 2017. Os critérios de inclusão foram prontuários de pacientes acima de 18 anos e com úlceras de membros inferiores. Resultados:A população contemplou 168 prontuários dos quais apenas 53 serviram para análise. Os dados foram analisados utilizando a estatística descritiva e inferencial pelo Teste Exato de Fisher. Conclusão:A média de idade foi de 61.55 anos, a maioria dos participantes eram homens, pardos, casados, com ensino fundamental incompleto, aposentados e hipertensos. Houve uso de fármacos em $100 \%$ dos pacientes e significância na associação das complicações sobrepeso com necrose $(0,044)$, sexo com odor $(0,043)$ e raça com amputação $(0,040)$. Espera-se que a presente pesquisa sirva de norte para outros pesquisadores e no subsídio de uma melhor assistência.
\end{abstract}

Palavras-chave: Perfil de saúde; Úlcera da perna; Ferimentos e lesões; Enfermagem.

\begin{abstract}
Objective:To describe the sociodemographic and clinical profile of patients admitted with lower limb ulcers in a teaching hospital; identify the main clinical and surgical procedures performed to treat these patients and relate the risk factors and complications found in these wounds. Method:This is a documentary-descriptive research with a quantitative approach, developed in a teaching hospital, in the city of Campina Grande-PB, through the analysis of medical records of patients who were hospitalized in medical clinics, in June 2012 to March 2017. Inclusion criteria were medical records with patients over 18 years and with lower limb ulcers. Results: The population included 168 medical records, of which only 53 served for analysis. Data were analyzed using descriptive and inferential statistics by Fisher's Exact Test. Conclusion:The average age was 61.55 years, most participants were men, brown, married, with incomplete elementary school, retired and hypertensive. Drugs were used in $100 \%$ of the patients and significant in the association of overweight complications with necrosis $(0.044)$, sex with odor $(0.043)$ and race with amputation (0.040). It is hoped that the present research will serve as a guide for other researchers and for better assistance.
\end{abstract}

.Key-words: Health profile; Leg ulcer;Wounds and injuries; Nursing.

NOTA

I Bacharel em Enfermagem pela Universidade Federal de Campina Grande (UFCG). Professor do Curso Técnico em Enfermagem do Instituto Técnico do Brasil (ITEC-BR), Polo Assú-RN. Enfermeiro da Urgência e Emergência do Centro de Saúde Rosália Gondim de Melo, Paraú-RN. Membro do grupo de estudos e Pesquisas interdisciplinares em saúde e enfermagem (GEPISE/UFCG). Especialização em andamento em Enfermagem do Trabalho pela Faculdade Venda Nova do Imigrante (FAVENE). Email: Belmirojr10@hotmail.com

2 Doutora em Enfermagem pela Universidade Federal da Paraíba (UFPB). Mestre em Enfermagem pela UFPB. Mestre em Enfermagem em Terapia Intensiva pela Sociedade Brasileira de Terapia Intensiva (SOBRATI - Mestrado Profissionalizante). Especialista em Enfermagem do Trabalho pela Faculdade Integrada de Patos - FIP. Graduada em Enfermagem pela UFPB. Docente do curso de graduação em enfermagem da Universidade Federal de Campina Grande, Campus Cuité. Membro do Grupo de Estudos e Pesquisa em Tratamento de Feridas (GEPEFE)/UFPB e do Grupo de Estudos e Pesquisas Interdisciplinares em Saúde e Enfermagem (GEPISE/UFCG). Especialização em andamento em Enfermagem Dermatológica, pela Sociedade Brasileira de Enfermagem em Feridas e Estética em parceria com a Estácio de Sá. Email:alanatamar@gmail.com

3 Doutora em Enfermagem. Professora da Universidade Federal de Campina Grande, campus Cuite-PB. E-mail: lidiane.lima@ufcg.edu.br

4 Enfermeiro (FSM). Especialista em Saúde da Família (FSM). Mestre em Enfermagem (UFPB). Doutor em Saúde Coletiva (UFRN). Professor Adjunto da Universidade Federal de Campina Grande, Campus Cuité. Email: matheusnogueira.ufcg@gmail.com 


\section{INTRODUÇÃO}

As feridas representam um grande desafio à nível mundial e no cenário brasileiro, principalmente pelo ônus que acarretam e pela alta morbimortalidade desde às feridas agudas que complicam, quanto às que já são crônicas devido a algum problema associado à saúde do paciente.

Assim, no que diz respeito ao tempo de surgimento, as feridas classificam-se em agudas e crônicas. As primeiras cicatrizam dentro do período esperado e são oriundas de traumas ou cirurgias e traz-se um bom feedback ao tratamento de escolha enquanto as crônicas apresentam adversidades que dificultam sua cicatrização durante a terapêutica escolhida'.

Um bom exemplo das injúrias teciduais caracterizadas como crônicas, são as úlceras de membros inferiores - MMII, pois acometem pessoas de todo o mundo num percentual de I a I,5\% e localizam-se abaixo do joelho. Como fatores contribuintes para o surgimento dessas feridas, podem-se citar diversas causas como é o caso de agravos vasculares, neuropáticos, linfedemas, artrites reumatoides, traumas, osteomielites crônicas, anemia falciforme, vasculites, tumores cutâneos, infecções crônicas, idade superior a 69 anos dentre outras².

No tocante às úlceras de MMII, merecem destaque as úlceras vasculogênicas, que podem ser arterial ou venosa, e têm origem devido à insuficiência das artérias e das veias, respectivamente.Atingem um elevado índice $(70 \%)$ de pessoas que apresentam úlceras de perna, além de também poderem se apresentar na forma mista ${ }^{3}$. Pesquisa realizada em um ambulatório de feridas, num hospital universitário, no Brasil, identificou que $51 \%$ dos participantes apresentavam úlcera venosa e $2 \%$ úlcera de etiologia arterial ${ }^{4}$.

Também considerada uma das causas de úlceras de MMII, a erisipela, por sua vez, é uma das doenças que mais causa linfedema e, quando progride sem tratamento adequado, evolui para o surgimento de lesões dolorosas. Apresenta recidiva em sua maioria e é caracterizada por uma infecção aguda cutânea acometida, essencialmente, por bactérias de etiologia estreptocócica. Por ser uma celulite superficial, compromete o plexo linfático subjacente e apresenta-se em placas eritematosas na superfície da pele com edema, dor, calor e limitações demarcadas $^{5-6}$. Pesquisa realizada mostra que apesar da erisipela ser um problema assíduo na saúde, poucos são os estudos que abordam sobre sua epidemiologia, entretanto, essa mesma pesquisa aponta incidência de 0,2/1000 pessoas/ano a 24,6/1000 pessoas/ano em regiões distintas, sendo os mais acometidos indivíduos de 60 a 80 anos e maior prevalência no público feminino ${ }^{5}$.

Outro tipo de lesão de MMII muito frequente ocorre em pacientes com diabetes mellitus, doença crônica não transmissível de caráter multifatorial. Denomina-se pé diabético, a destruição ou ulceração dos tecidos mais profundos do pé relacionadas às neuropatias sensitiva, motora e autonômica ou doenças arteriais associadas ao diabetes mellitus. $O$ pé diabético tornou-se responsável por $70 \%$ das amputações nos adultos em todo o mundo ${ }^{7}$.

Menos comum, mas também considerada uma ferida de MMII, a úlcera hipertensiva de Martorell foi observada pela primeira vez em 194I por Hines e em seguida, em 1945, por Martorell. Caracteriza-se como uma úlcera arredondada, com extensão de 2 a $4 \mathrm{~cm}$, com tecidos granulosos ou necróticos, extremamente dolorosa, mais comum nas mulheres na faixa etária entre os 50 e 60 anos de vida, geralmente localizada no terço distal de MMII, apresenta dor desproporcional ao seu tamanho e o paciente tem, em seu histórico de cronicidade, hipertensão arterial sistêmica grave de longa permanência assim como também o diabetes mellitus tipo $2^{8}$.

Diante da problemática apresentada, as úlceras de MMIl podem ser de diferentes etiologias, acarretam gastos não planejados que cada paciente enfrenta com sua terapêutica, além das complicações que vão surgindo, desproporcionais à qualidade de vida.A família de cada paciente e - Sistema Único de Saúde, também estão envolvidos com o ônus dessa problemática e com o seu impacto negativo. É frequente que pessoas com úlcera sejam reclusas da sociedade pelo estigma enfrentado, pela falta de mobilidade, pela capacidade funcional reduzida e pelas diversas internações hospitalares decorrentes de infecções e necroses ${ }^{3}$.

Nesta perspectiva, compreende-se a significativa relevância desta pesquisa, uma vez que a realidade de pacientes que fazem tratamento de úlceras crônicas de MMII é mascarada com a subnotificação dos gastos enfrentados com a terapêutica, além das inúmeras internações decorrentes da progressão do estado clínico, da falta de educação em saúde e do acesso ao serviço que contribuam na prevenção dos agravos.Além disso, poderá servir de norte para que pesquisadores, enfermeiros e gestores identifiquem as lacunas existentes e adotem novas medidas a fim de minimizar a onerosidade enfrentada por cada sujeito envolvido, através da obtenção de resultados satisfatórios no menor tempo possível e com o menor custo.

O presente trabalho teve como objetivos descrever o perfil sociodemográfico e clínico de pacientes internados com úlceras de MMII em um hospital escola; identificar os principais procedimentos clínicos e cirúrgicos realizados para o tratamento desses pacientes; e, por fim, relacionar os fatores de risco e as complicações encontradas nessas feridas.

\section{MÉTODOS}

Trata-se de uma pesquisa do tipo documental-descritiva, com abordagem quantitativa, desenvolvido no Serviço de Arquivo Médico e Estatístico (SAME) de um hospital escola localizado em Campina Grande/PB, a partir da leitura de prontuários de pacientes internados 
nas Clínicas Médicas feminina e masculina, considerando o intervalo entre junho de 2012 e agosto de 2017 . Os critérios de inclusão foram prontuários que pertencessem a pacientes acima de 18 anos e com úlcera de MMII e, como critérios de exclusão, prontuários inelegíveis e diagnóstico indefinido.

Realizou-se um levantamento dos pacientes internadas nas referidas clínicas durante os cinco anos que correspondiam ao objetivo da pesquisa através do Código Internacional de Doenças - CID para se obter a amostra. Foram utilizados os CID's: 183 - varizes dos MMII; 183.0 - varizes dos MMII com úlcera; 183.2 - varizes dos MMII com úlcera e inflamação; 183.9 - varizes dos MMII sem úlcera ou inflamação; L89.9 - úlcera de decúbito; L97.7 - úlcera dos MMII não classificada em outra parte, e L98.4 - úlcera crônica de pele não classificada em outra parte, totalizando 165 prontuários. Contudo, foram excluídos 112 documentos por não estarem de acordo aos critérios de inclusão, o que resultou em um total de 53 prontuários para análise.

Como instrumento desta pesquisa foi utilizado um roteiro estruturado contendo itens relacionados aos dados sociodemográficos, clínicos e terapêuticos de pacientes com úlceras de MMII, construído a partir da literatura pertinente, de modo a atender aos objetivos propostos pela pesquisa.

Depois da aprovação da pesquisa pelo Comitê de Ética em Pesquisa (CEP), sob CAEE $n^{\circ} 2.514 .070$, os prontuários foram solicitados ao SAME, conforme agendamento pelo próprio serviço.

O software Excel foi utilizado na construção de uma planilha de dados para as questões contidas nos instrumentos de coleta dos dados e as informações foram transferidas para o pacote estatístico SPSS (Statistical Package for Social Sciences) - versão 21.0.

Para análise dos dados, foram obtidas distribuições absolutas e relativas, além dos cruzamentos das variáveis das complicações das feridas com o perfil sociodemográfico e clínico dos participantes, por meio do Teste Exato de Ficher, considerado estatisticamente significativo quando o $\mathrm{p}$-valor $\leq 0,05$.

\section{RESULTADOS}

Os prontuários foram coletados na seguinte distribuição de anos: 06 (II,3\%) em 20I2; 03 (5,7\%) em 20।3; 08 (I5, I\%) em 20I4; I3 (24,5\%) em 20I5; I7 (32, I\%) em 2016 e 06 (II,3\%) em 2017.

Os seguintes dados foram investigados e discutidos: perfil sociodemográfico e clínico; procedimentos clínicos e cirúrgicos; fatores de risco e complicações nas feridas dos participantes.

\section{Descrição do perfil sociodemográfico e clínico de pacientes com úlceras de MMII}

A idade dos pacientes variou de 18 a 98 anos, sendo a média de idade de 61,55 anos e o desvio padrão de 16,88 .

A Tabela 01 exibe outros dados referentes ao perfil sociodemográfico dos pacientes.

Conforme a Tabela 0I, dos 53 pacientes, a maioria $(67,9 \%)$ era do sexo masculino, da raça parda $(66 \%)$ e estado civil casado $(39,6 \%)$. No que se refere à escolaridade, 29 (54,7\%) prontuários estavam subnotificados, com prevalência do ensino fundamental incompleto para os que constavam esse registro.A maior parte correspondia a aposentados $(37,7 \%)$.

$\mathrm{Na}$ Tabela 02 estão apresentados os dados relacionados às doenças dos pacientes.

A respeito das doenças, a maioria dos pacientes eram hipertensos, $32(60,6 \%)$, seguido dos pacientes com diabetes, 17 (32, $1 \%)$.

Na Tabela 03 está a apresentação dos tipos de úlceras de MMII.

Conforme tabela apresentada, a maioria, 37 (69,8\%), possuía úlcera de etiologia venosa, obtendo segundo lugar na tabela a úlcera após episódio de erisipela, com I0 (18,9\%) pacientes, úlcera pé diabético vem logo em seguida, com um total de 07 (I3,2\%) pacientes.

\section{Identificação dos principais procedimentos rea- lizados para o tratamento de pacientes internados com úlcera de MMII}

Os achados obtidos revelam que todos (100\%) os pacientes faziam tratamento farmacológico no período da hospitalização - principalmente antibiótico, analgésicos, corticoides, anticoagulantes, antifúngicos, antidiabéticos e anti-hipertensivos, desses, 24 (45,3\%) pacientes também realizaram tratamento não farmacológico do tipo: dietas - hipossódicas, hipoglicêmica e meias compressivas e apenas I3 $(24,5 \%)$ realizaram tratamento cirúrgico desbridamentos e amputações.

\section{Relação dos fatores de risco com as complicações em feridas presentes nos pacientes com úlceras de MMII}

A Tabela 04 aponta as complicações das feridas dos pacientes com úlceras de MMII durante a hospitalização.

Ao observar aos dados da Tabela 04, é perceptível que $22(41,5 \%)$ dos pacientes apresentaram infecção durante a hospitalização, 17 (32, I\%) dos casos foram acompanhados de dor, 8 (I5,I\%) dos pacientes apresentaram odor nas suas lesões, assim como também essa mesma proporção apresentaram necrose como tecido desvitalizado e $3(5,7 \%)$ pacientes amputaram o membro afetado.

A Tabela 05 traz a relação das complicações das feridas com os fatores sociodemográficos e clínicos durante a hospitalização de pacientes com as úlceras de MMII. 
TABELA 01 - Dados sociodemográficos de pacientes com úlceras de MMII de um Hospital Escola. Campina Grande, PB, Brasil, 2018.

\begin{tabular}{|c|c|c|}
\hline Dados Sociodemográficos & $\mathbf{F}$ & $\%$ \\
\hline $\begin{array}{ll} & \text { Sexo } \\
\text { Masculino } & \\
\text { Feminino }\end{array}$ & $\begin{array}{l}36 \\
17\end{array}$ & $\begin{array}{l}67,9 \\
32,1\end{array}$ \\
\hline $\begin{array}{ll} & \text { Raça } \\
\text { Parda } & \\
\text { Não informado } \\
\text { Branco } \\
\text { Negro }\end{array}$ & $\begin{array}{l}35 \\
8 \\
7 \\
3\end{array}$ & $\begin{array}{c}66 \\
15,1 \\
13,2 \\
5,7\end{array}$ \\
\hline $\begin{array}{l}\text { Casado Civil } \\
\text { Solteiro } \\
\text { Viúvo } \\
\text { Não informado } \\
\text { Divorciado } \\
\text { Separado }\end{array}$ & $\begin{array}{l}21 \\
18 \\
7 \\
4 \\
2 \\
1\end{array}$ & $\begin{array}{c}39,6 \\
34 \\
13,2 \\
7,5 \\
3,8 \\
1,9\end{array}$ \\
\hline $\begin{array}{l}\text { Escolaridade } \\
\text { Não Informado } \\
\text { Ens. Fund. Incompleto } \\
\text { Não Escolarizado } \\
\text { Ens. Med. Completo } \\
\text { Ens. Fund. Completo } \\
\text { Ens. Med. Incompleto } \\
\text { Ens. Sup. Completo }\end{array}$ & $\begin{array}{l}29 \\
11 \\
6 \\
3 \\
2 \\
1 \\
1\end{array}$ & $\begin{array}{l}54,7 \\
20,8 \\
11,3 \\
5,7 \\
3,8 \\
1,9 \\
1,9\end{array}$ \\
\hline $\begin{array}{l}\text { Profissão/Ocupação } \\
\text { Aposentado } \\
\text { Agricultor } \\
\text { Empregado } \\
\text { Autônomo } \\
\text { Do Lar } \\
\text { Não Informado } \\
\text { Desempregado } \\
\text { Estudante }\end{array}$ & $\begin{array}{l}20 \\
10 \\
8 \\
4 \\
4 \\
3 \\
3 \\
1\end{array}$ & $\begin{array}{l}37,7 \\
18,9 \\
15,1 \\
7,5 \\
7,5 \\
5,7 \\
5,7 \\
1,9\end{array}$ \\
\hline
\end{tabular}

Fonte: Dados da pesquisa.

TABELA 02 - Dados de doenças dos pacientes com úlceras de MMIl de um Hospital Escola. Campina Grande, PB, Brasil, 2018.

\begin{tabular}{l|c|c}
\hline \multicolumn{1}{c|}{ Doenças de Base } & F & \% \\
\hline Hipertensão Arterial Sistêmica & 32 & 60,4 \\
\hline Diabetes & 17 & 32,1 \\
\hline Outras Doenças* & 16 & 30,6 \\
\hline Insuficiência Venosa & 13 & 24,5 \\
\hline Sobrepeso & 2 & 3,8 \\
\hline
\end{tabular}

*Outras doenças identificadas: insuficiência cardíaca crônica; insuficiência renal crônica; síndrome da imunodeficiência adquirida; constipação intestinal crônica; artrite reumatoide; acidente vascular encefálico; cardiopatia; hipotireoidismo; alergia; doença arterial obstrutiva periférica. **Questão de múltiplas respostas

Fonte: Dados da pesquisa.

TABELA 03 - Tipos de úlceras de MMII de pacientes de um Hospital Escola. Campina Grande, PB, Brasil, 2018.

\begin{tabular}{l|c|c}
\hline Tipo de Úlcera Vasculogênica & F & $\%$ \\
\hline Úlcera Venosa & 37 & 69,8 \\
\hline Úlcera Erisipela & 10 & 18,9 \\
\hline Úlcera Pé Diabético & 7 & 13,2 \\
\hline Úlcera Martorell & 3 & 5,7 \\
\hline Úlcera Vasculogênica & 2 & 3,8 \\
\hline Úlcera Arterial & 1 & 1,9 \\
\hline
\end{tabular}

${ }^{*}$ Questão de múltiplas respostas. Fonte: Dados da pesquisa 
TABELA 04 - Complicações das úlceras de MMII de pacientes durante o desfecho hospitalar de um Hospital Escola. Campina Grande, PB, Brasil, 2018.

\begin{tabular}{l|c|c}
\hline \multicolumn{1}{c|}{ Complicações das Úlceras de MMII } & $f$ & $\%$ \\
\hline Infecção & 22 & 41,5 \\
\hline Dor & 17 & 32,1 \\
\hline Odor & 8 & 15,1 \\
\hline Necrose & 8 & 15,1 \\
\hline Amputação & 3 & 5,7 \\
\hline
\end{tabular}

*Questão de múltiplas respostas.

Fonte: Dados da pesquisa.

TABELA 05 - Relação das complicações das feridas com o perfil sociodemográfico e clínico durante a hospitalização de pacientes com úlceras de MMII de um Hospital Escola. Campina Grande, PB, Brasil, 2018.

\begin{tabular}{l|c|c|c|c|c}
\hline & $\begin{array}{c}\text { INFECÇÃO } \\
\text { p-valor }\end{array}$ & $\begin{array}{c}\text { NECROSE } \\
\text { p-valor }\end{array}$ & $\begin{array}{c}\text { DOR } \\
\text {-valor }\end{array}$ & $\begin{array}{c}\text { ODOR } \\
\text {-valor }\end{array}$ & $\begin{array}{c}\text { AMPUTAÇÃO } \\
\text {-valor }\end{array}$ \\
\hline Idade & 0,891 & 0,856 & 0,724 & 0,131 & 0,102 \\
\hline Sexo & 0,862 & 0,650 & 0,066 & $0,043^{*}$ & 0,863 \\
\hline Raça & 0,985 & 0,718 & 0,275 & 0,973 & $0,040^{*}$ \\
\hline Escolaridade & 0,638 & 0.132 & 0,355 & 0,084 & 0,296 \\
\hline Ocupação & 0,346 & 0,471 & 0,108 & 0,101 & 0,200 \\
\hline Diabetes & 0,633 & 0,436 & 0,619 & 0,895 & 0,184 \\
\hline Hipertensão Arterial Sistêmica & 1,000 & 0,348 & 0,326 & 0,781 & 1,000 \\
\hline Ins. Venosa Crônica & 0,256 & 0,244 & 0,927 & 0,594 & 0,116 \\
\hline Ins. Arterial & 1,000 & 0,358 & 1,000 & 1,000 & 0,405 \\
\hline Doença Auto Imune & 1,000 & 0,151 & 1,000 & 1,000 & 1,000 \\
\hline Sobrepeso & 0,661 & $0,044 *$ & 0,493 & 0,236 & 1,000 \\
\hline Desnutrição & 1,000 & 0,151 & 1,000 & 1,000 & 1,000 \\
\hline Outras Doenças & 0,077 & 0,246 & 0,642 & 0,724 & 1,000 \\
\hline Etilista & 0,300 & 0,660 & 0,946 & 0,883 & 0,452 \\
\hline Tabagista & 0,964 & 0,876 & 0,684 & 0,953 & 0,687 \\
\hline Sedentário & 0,605 & 0,212 & 0,472 & 0,085 & \\
\hline
\end{tabular}

${ }^{*}$ p-valor $\leq 0,05$ pelo Teste Exato de Fisher.

Fonte: Dados da pesquisa.

Conforme os dados apresentados, por meio do Teste Exato de Fisher observa-se que há associação significativa entre as variáveis sobrepeso com necrose ( $\mathrm{p}$-valor $=0,044)$, sexo com odor ( $p$-valor $=0,043)$, e raça com amputação ( $\mathrm{p}$-valor $=0,040$ ).

Quanto ao desfecho, todos os pacientes receberam alta hospitalar, levando a crer que a cura ou parcela dela foi obtida.

\section{DISCUSSÃO}

A média de idade de 61,55 anos e o sexo masculino predominante concordam com um estudo realizado em um serviço de angiologia ambulatorial também em um hospital universitário, em que os autores encontraram pacientes com média de idade de 60,9 anos e afirmam que os homens procuram os serviços de saúde apenas quando apresentam complicações da insuficiência vascular, como as úlceras, o que leva a aumentar o número de homens com problemas de saúde mais graves?.

A raça parda atingiu um maior percentual de pessoas, discordando de outra pesquisa desenvolvida com pacientes idosos com úlceras de perna, em que a maioria era da raça branca ${ }^{9}$. Quanto ao estado civil, a categoria casada alcançou maior taxa, conforme outro artigo científico, que traz o maior índice de acometimento em pessoas casadas ou em união estável ${ }^{10}$, o que pode ser explicado devido ao fato de que essas lesões aparecem mais comumente em pessoas idosas, com estabilidade da união entre o casal, como apresentado acima.

No que se refere à escolaridade, uma explicação plausível para o achado seria o fato da pesquisa ter acontecido em um hospital público de saúde, no qual é comum sua clientela apresentar baixa escolaridade e, consequentemente, menor poder aquisitivo, limitando sua procura por um serviço de saúde especializado e menos burocrático, que favorece a piora das lesões.

No viés da profissão ou ocupação, a maioria correspondia a aposentados, anuindo-se, assim, com o exposto na literatura de que essas lesões aparecerem geralmente em idosos ${ }^{10}$. Uma resposta plausível para os dados encontrados é que o envelhecimento deixa a pele mais enrugada, delgada e ressecada. Associada a esse fator obtêm-se um decréscimo na troca de oxigenação tecidual, maior desidratação e menor flexibilidade relacionada às fibras de colágeno que se tornam mais espessas, as fibras elásticas perdem sua elasticidade, além da redução da ca- 
mada de gordura no tecido subcutâneo, o que torna a pele mais vulnerável com a idade avançada". Além desses fatos que predispõem o aumento dos riscos para as lesões de pele, as comorbidades, desnutrição e imobilidade potencializam o surgimento de úlceras crônicas.

Com relação às doenças de base, obteve-se prevalência da hipertensão arterial sistêmica. Ao observar dados catalogados em trabalho científico publicado com pacientes com úlcera vasculogênica no Piauí, pôde-se perceber a consonância existente entre os dados da presente pesquisa com o trabalho analisado, apontando esse, maior percentual também na hipertensão arterial em pessoas com feridas crônicas ${ }^{10}$.

Dentre as várias lesões que podem surgir em MMII, nesta pesquisa houve prevalência da úlcera venosa, o que corrobora dados apresentados por outro estudo que aponta que o principal tipo de úlcera de MMII é de etiologia venosa e suas principais características são: situarse na região maleolar medial ou lateral, apresentarem-se grandes e exsudativas ${ }^{12}$.

No tocante ao tratamento, observa-se o uso de múltiplos medicamentos ou polifarmácia, comum em pessoas acima de 65 anos, utilizadas para o controle de infecção, problema recorrente em feridas crônicas, e controle de doenças de base, a exemplo de hipertensão e diabetes, doenças frequentes nesses pacientes ${ }^{13}$, conforme evidenciado na tabela 02. É importante frisar ainda, que o uso inadequado de alguns medicamentos pode retardar a cicatrização da ferida.

Vale ressaltar que, apesar da úlcera venosa predominar dentre os participantes da pesquisa, não se identificou o uso de medicações flebotômicas, a exemplo da diosmina e hesperidina, importantes para diminuir a permeabilidade capilar - ação anti-inflamatória por reduzir a adesividade de células de defesa sozinha e adjuvante, e podem ser utilizadas como coadjuvantes à terapia compressiva para redução dos sintomas da doença venosa crônica, como o edema ${ }^{14}$.

Ao sumarizar as informações obtidas com relação às complicações, nesta investigação, percebe-se a prevalência de infecção e dor. Outra fonte científica evidencia a correlação entre os achados, uma vez que $40 \%$ dos pacientes apresentaram indícios de infecção através do odor fétido. A pesquisa também mostrou que dos pacientes entrevistados, $20 \%$ relataram dor ${ }^{15}$. A infecção leva a ferida a retardar sua cicatrização já que os microrganismos competem com o oxigênio e nutrientes disponíveis no leito da lesão, liberam toxinas e com isso agridem o tecido sadio prolongando a fase inflamatória, com sinais flogísticos como a dor, e causando necrose.

A investigação da relação entre as complicações e o perfil sociodemográfico e clínico dos participantes apontou, por meio do Teste Exato de Fisher, que há associação das variáveis sobrepeso com necrose ( $\mathrm{p}$-valor $=0,044)$, sexo com odor ( $p$-valor $=0,043)$ e raça com amputação ( $p$-valor $=0,040$ ), conforme os dados dispostos na tabela 05 .

Os hábitos alimentares considerados inadequados são frequentes na população em geral e em pesquisa realizada com 36 pessoas com úlceras crônicas de MMII em tratamento no ambulatório de feridas apontou insuficiência venosa em $30,6 \%$, obesidade em $33,3 \%$ e aumento da circunferência abdominal em $75 \%$ dos entrevistados $^{16}$. Essa mesma investigação encontrou ingesta de nutrientes abaixo do recomendado, como vitamina A, C, zinco e proteínas e excesso da ingesta de ferro, o que pode acarretar dificuldade de cicatrização dessas lesões devido à falta de alguns nutrientes ou pelo excesso de outros.

Outrossim, o sobrepeso, a obesidade e aumento da circunferência abdominal dificultam o retorno venoso e, consequentemente, a cicatrização, em decorrência do comprometimento da circulação sanguínea e da hipoventilação que reduzem a perfusão de oxigênio e nutrientes do tecido, principalmente em pacientes com insuficiência venosa, causando aumento da hipertensão venosa e piora do processo inflamatório, o que pode estar também associado ao surgimento ou piora da necrose ${ }^{17}$.

Apesar da presente pesquisa apontar os homens como sendo o principal acometido por úlceras vasculogênicas, há estudo que mostra prevalência em mulheres ${ }^{18}$. Acredita-se, aqui, que o fator sexo masculino tem importância na significância dos resultados da cicatrização da ferida - no tocante ao odor, por ser o homem o mais susceptível a desenvolver úlceras vasculogênicas como informam os achados do presente trabalho. Não foram encontradas pesquisas que abordassem o fator sexo masculino com a complicação odor e sua relação.

Outro fator associado às complicações nas úlceras de MMII foi a raça com a amputação. Nesta pesquisa, 66,6\% da raça parda e 33,4\% dos negros sofreram amputação. Não houve amputação em pacientes da raça branca. Tal fator pode ser explicado através de dados de um estudo realizado em Juiz de Fora - MG, que aborda a visão da população negra - considerando pretos e pardos, segundo a Política Nacional de Saúde Integral da População Negra - PNSIPN, no qual esse grupo racial afirma dificuldades no acesso aos serviços de saúde. Além disso, os entrevistados também relataram discriminação nos serviços de saúde e isso leva à redução na procura das consultas médicas ${ }^{19}$.

Quanto ao desfecho, todos os pacientes receberam alta hospitalar, levando a crer que a cura ou parcela dela foi obtida. Contudo esses dados são divergentes de outros estudo que aponta um alto grau dos índices que interferem diretamente na morbimortalidade de pacientes com doenças crônicas não transmissíveis ${ }^{20}$. 


\section{CONCLUSÃO}

O presente estudo evidenciou através dos achados a identificação do perfil sociodemográfico e clínico dos pacientes internados com úlceras de MMII nas clínicas médicas feminina e masculina do Hospital Universitário Alcides Carneiro, nos quais, em sua maior partes os pacientes eram homens, idosos, pardos, casados, possuíam ensino fundamental incompleto e eram aposentados. Quanto às condições clínicas associadas, apresentavam morbidades como a hipertensão arterial, diabetes, insuficiência venosa e sobrepeso. A úlcera venosa foi maioria sobre as demais.

Todos os pacientes fizeram uso do tratamento farmacológico e apresentaram durante a internação infecção, dor, odor, necrose e realizaram amputação de MI. Houve relevância significativa na associação das variáveis sobrepeso com necrose, sexo com odor e raça com amputação.

Como limitações encontradas, pode-se citar a quantidade de prontuários diários fornecidas pelo SAME do hospi- tal - dez prontuários, prejudicando o andamento da coleta, além disso, também é valido ressaltar a ilegibilidade da caligrafia dos profissionais, principalmente médicos, divergência de informação e subnotificações nos prontuários.

Seria importante que os coordenadores, enfermeiros e médicos das referidas clínicas, pudessem organizar o serviço por meio de protocolos e fichas padronizadas, a fim de reduzir erros de registros e até mesmo a omissão de dados. Além disso, a educação permanente com os profissionais da assistência, com enfoque na admissão e evolução do paciente agregaria mais valor à assistência prestada, a fim de minimizar ou abolir as falhas encontradas.

Assim, espera-se que a presente pesquisa sirva de norte para outros pesquisadores da área, além de subsidiar o incentivo a práticas corretas de trabalho para enfermeiros, médicos, técnicos de enfermagem e também gestores, no vislumbre de uma assistência mais qualificada e com um sistema de informação mais plausível e eficaz para a prestação da assistência continuada. 


\section{REFERÊNCIAS}

I. Campos MGCA, Sousa ATO,Vasconcelos JMB. Fundamentos teóricos e avaliação de feida. In: Lucena SRP, Gomes SKA. Ferida complexa e estomias: aspectos preventivos e manejo clínico. João Pessoa. 2016. p 58-100.

2.Afonso A, et al. Úlcera crónica do membro inferior - experiência com cinquenta doentes. Angiol Cir Vasc [Internet]. 2013 [acessado em I4 de jun de 20I8]; 9(4): I48-I53. Disponível em: https://www.sciencedirect.com/science/article/ pii/SI646706XI370035 I

3. O'donnell Júnior TF, et al. Management of venous leg ulcers: clinical practice guidelines of the society for vascular surgery and the American Venous Forum. J Vasc. Surg [Internet]. 2014 [acessado em 10 de jun de 2018]; 60(2):3s-59s. disponível em: https://www.ncbi.nlm.nih.gov/ pubmed $/ 24974070$

4. Oliveira BGRB, Castro JBA, Granjeiro JM. Panorama epidemiológico e clínico de pacientes com feridas crônicas tratados em ambulatório. Rev. enferm, UERJ [Internet]. 2013 [acessado em 10 de jun de 2018]; I:6I2-7. Disponível em: http://www.facenf.uerj.br/v2 I nesp I/v2 l ela09.pdf

5. Dalal A, et al. Interventions for the prevention of recurrent erysipelas and cellulitis. Cochrane Database of Systematic Reviews [Internet]. 2017 [acessado em 26 de nov de 2018]; 6:1-70. disponível em: https://www.cochranelibrary.com/ cdsr/doi// 0.1002//465 I858.CD009758.pub2/epdf/full

6. Cruz RAO, Miranda EG, Santos EC, Ferreira MGMS, Santana RA. Abordagem e reflexões para o cuidado do cliente com erisipela. Rebes [Internet]. 2016 [acessado em 26 de nov de 2018]; 6(I):22-26. Disponível em: http://oaji.net/articles/2016/2628-I46I620160.pdf

7. Seguel G. ¿Por qué debemos preocuparnos del pie diabético? Importancia del pie diabético. Rev Med Chile [Internet]. 2013 [acessado em I5 de maio de 20 I8]; |4I:I464-|469. Disponível em: https://scielo.conicyt.cl/pdf/rmc/vl4lnII/ art I4.pdf

8. Pontes AAN, Macieira NFA, Sousa RJ. Úlcera de martorell: análise epidemiológica e clínica em pacientes portadores de diabetes mellitus tipo 2. RSC online [Internet]. 2017[acessado em 12 de setembro de 2018]; 6(4):5- I5. 2017. Disponível em: http://www.ufcg.edu.br/revistasaudeeciencia/index.php/RSC-UFCG/article/view/46I

9. Eberhardt RT, Raffetto JD. Chronic Venous Insufficiency. Chronic venous insufficiency. Rev. Circulation [Internet]. 2014 [acessado em 10 de jun de 20I8]; I30(4):333-346. Disponível em: https://www.ncbi.nlm.nih.gov/pubmed/25047584

10. Monte BKS, Moura ECC, Costa JP, Silva GRF,Lopes VCA. Qualidade de vida de pacientes com úlceras vasculogênicas em tratamento ambulatorial. Rev da Rede de Enf do Nordeste [Internet]. 2018 [acessado em II de maio de 2018]; 19: :e3286. Disponível em: http://www.redalyc.org/articulo. oa?id=324054783009

I I. Macedo MCA, Tenório CA. Tratamento de rugas: Uma re- visão bibliográfica sobre Carboxiterapia, Radiofrequência e Microcorrente. Visão universitária [Internet]. 2015 [acessado em 15 de jun de 2018]; 2(1):54-78. Disponível em: http://www.visaouniversitaria.com.br/ojs/index.php/home/ article/view/56

12. Tavares APC, Sá SPC, Oliveira BGRB, Sousa AI. Qualidade de vida de idosos com úlceras de perna. Escola Anna Nery Revista de Enfermagem [Internet]. 2017 [acessado em I4 de maio de 2018]; 2 I(4):I-9. Disponível em: http://www. redalyc.org/articulo.oa?id= $\mid 27752022030$

13. Pereira KG, et al. Polifarmácia em idosos: um estudo de base populacional. Ver. Bras. Epidemiol [Internet]. 2017 [Acessado em I5 de maio de 2018]; 20(2):335-344. Disponível em: https://www.scielosp.org/article/rbepid/2017. v20n2/335-344/en/

14. Bush R, Camerota A, Meisnner M, Raffeto JD, HahnSR, Freeman k. Recommendations for the medical management of chronic venous disease: The role of Micronized Purified Flavanoid Fraction (MPFF). Phlebology [Internet]. 2017 [acessado em 14 de maio de 20I8]; 32(IS):319. Disponível em:http://journals.sagepub.com/doi/ abs/I0. I I77/02683555 I 769222 I

I5. Silva APR, et al. USO TERAPÊUTICO DA POMADA DE PRÓPOLIS EM DIFERENTES FERIDAS CRÔNICAS. Revista Perspectivas Online: Biológicas \& Saúde [Internet]. 2017 [acessado em 13 de junho de 20I8]; 7(24):40-46. Disponível em: http://www.seer.perspectivasonline.com.br/index. php/biologicas_e_saude/article/view/I 160

16. Dal Santos M, Franco S, Sanches LFZ, Rosalva R, Hack J, Magro NMM. Caracterização Nutricional de Pacientes com Úlceras Crônicas de Membros Inferiores em Tratamento no Ambulatório de Feridas do Campus Cedeteg da UNICENTRO, Guarapuava-PR. Journal of Health Sciences [Internet]. 2015 [acessado em I4 de junh de 2018]; I7(I): I-7. Disponível em: http://www.pgsskroton.com.br/seer/index. php/JHealthSci/article/view/3 /8/298

17. Perrone F, Paiva AA, Letícia SLMI, Faria CS, Paese MCS, Nascimento JEA et al. Estado nutricional e capacidade funcional na úlcera por pressão em pacientes hospitalizados. Rev. Nutr. [internet]. $201 \mathrm{I}$ [acessado em 25 de nov de 2018]; 24(3):431-42. Disponível em: http://www.scielo.br/scielo.php?script=sci_arttext\&pi$d=S|4| 5-527320 \mid$ I 000300006 .

18. Targino IG, Souza JSO, Santos NMG, Davim RMB, Silva RAR. Fatores relacionados ao desenvolvimento de úlceras em pacientes com Diabetes Mellitus. Rev Fund Care Online [Internet]. 2016 [acessado em I3 de maio de 2018]; 8(4):4929-4934. Disponível em: http://dx.doi. org/l0.9789/2I75-536I.20I6.v8i4.4929-4934

19. Chehuen Neto JA, et al. Política Nacional de Saúde Integral da População Negra: implementação, conhecimento e aspectos socioeconômicos sob a perspectiva desse segmento populacional. Rev. Saúde Pública [Internet]. 2015 
[acessado em 12 setembro de 2018]; 20(6):1909-16. Disponível em: http://www.scielo.br/pdf/csc/v20n6//4I3-8I23csc-20-06-1909.pdf

20. Barreto MS, Carreira L, Marcon SS. Envelhecimento populacional e doenças crônicas: Reflexões sobre os desafios para o Sistema de Saúde Pública. Revista Kairós Geron- tologia [Internet]. 2015 [acessado em I3 de jun de 20I8]; I8(I):325-339.disponível em: https://revistas.pucsp.br/index.php/kairos/article/view/26092

Recebido: 2019-12-30 Aceito: 2020-04-16 